\title{
Recent Advances in the Management of Metastatic Neck Cancer
}

\author{
${ }^{1}$ Adnan Darr, ${ }^{2}$ Somiah Siddiq
}

\begin{abstract}
One of the most important prognostic factors in head and neck cancer is the presence or absence, level, and size of metastatic neck disease.

Controversies in neck management still exist due to the paucity of quality clinical trials with most evidence extrapolated from retrospective studies. However, recent evidence has emerged to address some of these areas including the ideal management of the NO neck, the role of sentinel node biopsy in occult neck disease, posttreatment surveillance with positron emission tomography and computed tomography (PET-CT) and incorporation of $\mathrm{P} 16$ status and extranodal extension (ENE) in the recent 8 th edition of AJCC in nodal staging.
\end{abstract}

This paper provides an update on the current management of metastatic neck disease in the setting of squamous cancers arising from the upper aerodigestive tract.

Keywords: Chemotherapy, Computed tomography, Head and neck squamous cell cancer recurrent, Metastatic, Neck, Positron emission tomography, Radiotherapy.

How to cite this article: Darr A, Siddiq S. Recent Advances in the Management of Metastatic Neck Cancer. Int J Head Neck Surg 2018;9(2):61-70.

Source of support: Nil

Conflict of interest: None

\section{INTRODUCTION}

Head and neck squamous cell carcinoma (HNSCC) is the 8th most common cancer worldwide within the male population, with an increasing trend also noted amongst females. ${ }^{1}$ Tumors of the head and neck have a great propensity to metastasize to cervical nodes. The presence of neck nodes, coupled with their size and site play a crucial factor in the overall prognosis of HNSCC. ${ }^{2}$ Until recently,

\footnotetext{
${ }^{1}$ Registrar, ${ }^{2}$ Consultant

${ }^{1}$ Department of ENT, New Cross Hospital, Royal Wolverhampton Hospitals NHS Trust, Wolverhampton, West Midlands, UK

${ }^{2}$ Department of ENT, Head and Neck Unit, Heartlands Hospital, University Hospitals Birmingham NHS Foundation Trust Birmingham, West Midlands, UK
}

Corresponding Author: Somiah Siddiq, Head and Neck Consultant, Department of ENT, Head and Neck Unit, Heartlands Hospital, University Hospitals Birmingham NHS Foundation Trust Birmingham, West Midlands, UK. e-mail: somiah.siddiq@heartofengland.nhs.uk much controversy existed regarding the treatment of HNSCC due to the scant quantity of high-level data available to clinicians. The recent publication of randomized control trials (RCTs), as well as systematic reviews, has moved some way towards addressing some of the controversies in the management of metastatic neck disease.

\section{ASSESSMENT AND STAGING}

Approximately 150 lymph nodes are present on either side of the neck, with normal sizes ranging from $3 \mathrm{~mm}-3 \mathrm{~cm}$, although most are $<1 \mathrm{~cm}$. The largest node, often referred to as the jugulodigastric node (JDN), is found in level II situated within a triangle formed by the IJV, facial vein and posterior belly of the digastric muscle. The JDN receives lymph from a large region of the neck including the submandibular region, oropharynx and oral cavity. It is crucial for clinicians to appreciate that contralateral nodal spread may occur early in tumours that either lie in or adjacent to the midline. The Memorial SloanKettering group first published the widely adopted levels within the neck, containing lymph node groups, which represented the primary sites for metastases from HNSCC. A total of 6 neck levels were described, with level VII describing a chain of paratracheal nodes from the level of the suprasternal notch to the innominate artery. Further revisions included subdivisions of levels I, II and V (Table 1). Nodal staging should be radiologically confirmed and documented according to the TNM classification, prior to any medical or surgical intervention. Whilst imaging remains a key modality in the initial diagnosis, up to $33 \%$ of patients presenting with SCC of the oral cavity have occult cervical disease undetectable by conventional techniques (computed tomography (CT), magnetic resonance imaging (MRI) and positron emission tomography (PET). ${ }^{3}$ Liao et al. undertook a meta-analysis, comparing the diagnostic accuracy of various imaging modalities, including $\mathrm{CT}$, MRI, PET and US, when evaluating NO HNSCC patients. Of the 168 relevant articles which were identified, 7 studies fulfilled the inclusion criteria for CT, 6 for MRI, 11 for PET and 8 studies for USS respectively. The study reported no variation in sensitivity and specificity among the imaging modalities, with the exception of $\mathrm{CT}$, which had a superior specificity compared to USS. ${ }^{4}$ 
Table 1: Anatomical levels of the neck and boundaries

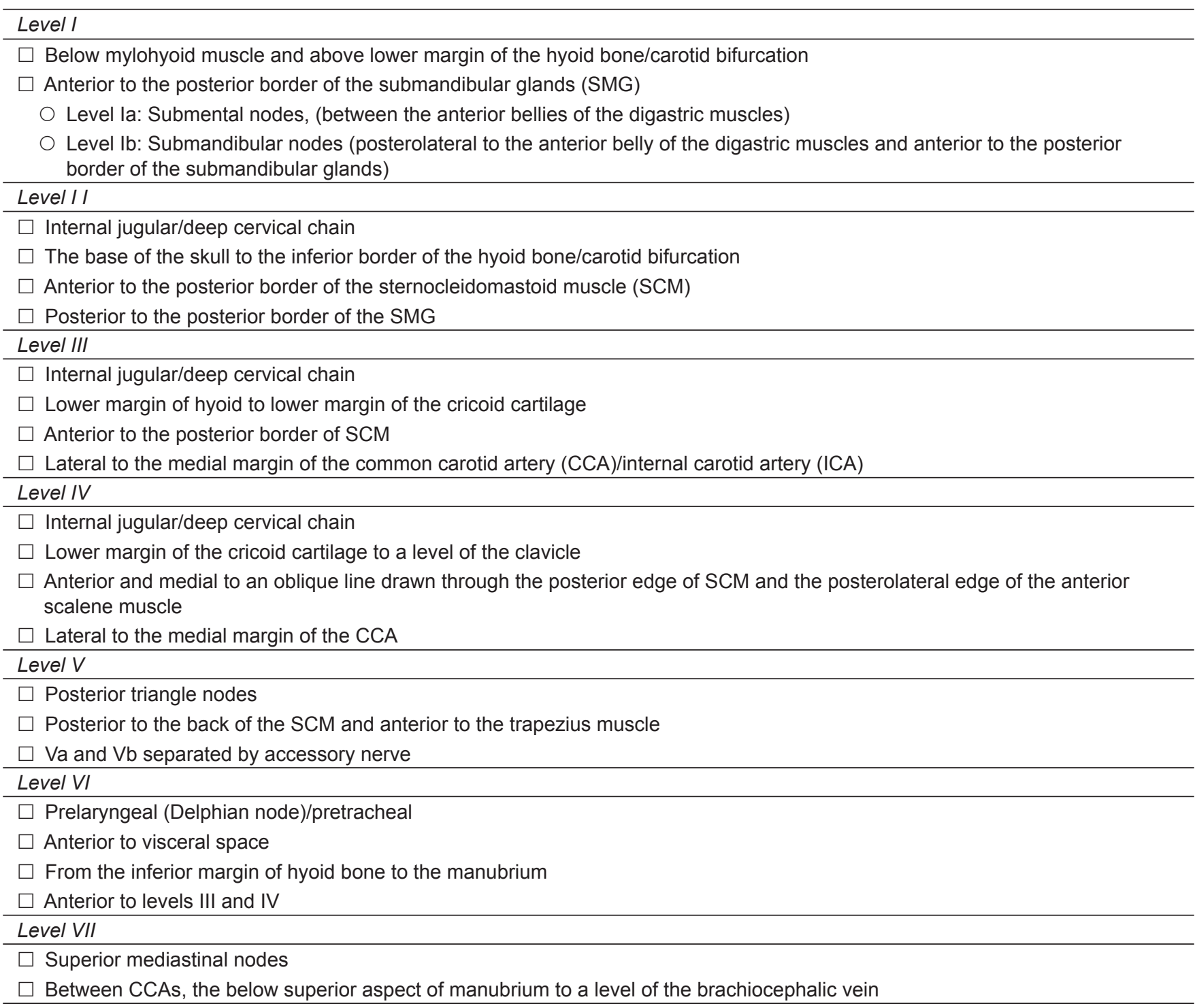

\section{Clinical Palpation}

Clinical palpation is an entirely operator dependent firstline method for assessment and follows an experiential learning curve. With sensitivity and specificity of $70-80 \%$, palpation is regarded as an inaccurate method of detecting cervical metastases, due in part to a variety of factors including; operator variability, the variability of overlying and surrounding soft tissue (such as post-radiotherapy changes), and size of nodal metastasis. ${ }^{2,5}$ Furthermore, in particular reference to the retropharynx, some areas are inaccessible and therefore cannot be clinically assessed.

\section{Computed Tomography and Magnetic Resonance Imaging}

Computed tomography (CT) as a modality in detecting cervical metastasis, has been routinely used since the early 1980s. ${ }^{6}$ CT boasts a higher diagnostic accuracy in detecting metastatic neck disease when compared to clinical examination. A meta-analysis comparing $\mathrm{CT}$ versus clinical examination following 647 neck dissections, elicited a sensitivity of $83 \%$ and specificity and overall accuracy of $83 \%$ in CT. ${ }^{7}$ Furthermore, positron emission tomography (PET), combined with CT can change the initial disease staging in up to 30\% of patients, ${ }^{2}$ with a far superior sensitivity in detecting occult primaries, synchronous primaries and distant disease progression. ${ }^{2}$

Magnetic resonance imaging (MRI) protocols include; T1/2 sequences, STIR, TIRM, DWI, perfusion +/- contrast, enabling detection of small lesions, whilst simultaneously permitting assessment of local tumor infiltration, and aiding operative planning. MRI demonstrates sensitivities of $82 \%$ and a specificity of $66.7 \%$ in determining soft tissue and bone/ marrow infiltration. MRI is comparable to CT in terms of sensitivity and specificity, although MRI demonstrates a higher sensitivity with respect to soft tissue assessment. ${ }^{8}$ 
Ultrasound Scan (USS) and Fine Needle Aspiration Cytology (FNAC) or Core Biopsy (CB)

A high spatial resolution, ease of multi-planar scanning, power Doppler, and the ability to undertake guided FNAC give USS a significant advantage over other imaging modalities when assessing for nodal disease in HNSCC. ${ }^{9}$ Ultrasound has demonstrated sensitivities of $87 \%$ in the diagnosis of metastatic neck cancer. The modality does, however, require both expertise and experience, and when these criteria have been achieved, high specificities of $98 \%$ have been reported. ${ }^{2}$ The disadvantages include, operator dependency, and an inability to visualize structures beyond bony and air interfaces due to the inability of sound waves to transmit through such mediums. ${ }^{10}$ Despite its widespread use in clinical practice, USS is yet to provide conclusive differentiation between benign and malignant disease. FNAC remains particularly useful in the assessment of palpable lymphadenopathy in the carcinoma of unknown primary (CUP) setting. A core biopsy provides the additional benefit over FNAC of providing tissue which can be subjected to immunohistochemical analysis, including isolation of HPV or Epstein-Barr virus (EBV) transcripts, raising the suspicion of a primary site within the oropharynx or nasopharynx respectively. Accuracy rates of FNAC are reported to be as high as $90 \%{ }^{11}$

Sonoelastography is a relatively novel modality, which has demonstrated some promise in the evaluation of neck nodes. ${ }^{12}$ Low-amplitude and frequency shear waves are propagated through tissue, with real-time Doppler used to image the resulting vibration pattern. The reduction in the amplitude of vibration as a result of tumor inhomogeneity is subsequently measured.

\section{Positron Emission Tomography and Computed Tomography}

This modality combines 18 F-fluorodeoxyglucose PET (18F-FDG PET) and CT to highlight both structural and metabolic changes within tumors and is a reliable modality in assessing response to treatment. ${ }^{8}$ Unfortunately, PET-CT is relatively nonspecific, with tracer uptake also demonstrated within inflammatory pathology. ${ }^{10}$ Negative predictive values of $94.5 \%$ and $96 \%$ have been demonstrated through meta-analyses of predominantly small cohort single-center studies in patients with HNSCC. ${ }^{13,14}$ Kyzas et al. aimed to assess the diagnostic accuracy of 18F-FDG PET in detecting lymph node metastases in patients with HNSCC through a meta-analysis, whilst also providing comparisons against conventional modalities. Across 32 studies [ $(n=$ 1236), 18F-FDG PET sensitivity was $79 \%$ ( $95 \%$ confidence interval CI $=72-85 \%$ ), with a specificity of $86 \%$ (95\% CI $=83-89 \%)$. In the case of cN0 patients, the sensitivity of 18F-FDG PET was remarkably low at $50 \%(95 \% \mathrm{CI}=37-63 \%)$, although the specificity was $87 \%$ (95\% CI $=76-93 \%)$. Where both 18F-FDG PET and conventional modalities were utilized, the sensitivity and specificity of 18 F-FDG PET was 80 and $86 \%$, and $75 \%$ and $79 \%$, respectively for the conventional modalities. ${ }^{15}$ Calls to implement PET-CT in routine practice are not only to aid in staging and planning, but to also exclude distant metastatic disease, which would otherwise be missed on conventional imaging, and invariably advocate a palliative approach.

\section{Open Biopsy}

Open biopsies are generally avoided as an initial diagnostic tool and only adopted in certain situations, such as unavailability of FNAC or core biopsy, equivocal/ nondiagnostic results, or when the clinical or radiological findings are consistent with a diagnosis of either lymphoma or anaplastic carcinoma. When all avenues have been exhausted in the assessment of a suspicious neck node, an open biopsy can be undertaken, namely a selective neck dissection, as there is no body of evidence to suggest that this alters the overall prognosis, should the correct treatment be instigated $<6$ weeks. ${ }^{16}$

\section{Treatment Options}

\section{Surgery}

Neck dissection has historically been the surgical treatment of choice in metastatic neck disease. The readily accepted neck dissection terminology produced by the American Academy of Otolaryngology and Head and Neck Surgery (1991), has been updated by the committee for Neck Dissection Classification of the American Head and Neck Society (Table 2). ${ }^{17}$ A trend now exists to categorize neck dissections into two distinct subdivisions; comprehensive (removal of levels I-V) and selective ( $<5$ levels). ${ }^{2,17}$ In an era

Table 2: Neck dissection classification

(1) Radical neck dissection (RND)

(2) Modified radical neck dissection

(3) Selective neck dissection

(4) Extended radical neck dissection
Removal of levels I-V, accessory nerve, IJV and SCM

Removal of levels I-V dissected; preservation of one or more of the accessory nerve, IJV or SCM (types I, II, III respectively)

Preservation of $\geq 1$ level of lymph nodes

Removal of $\geq 1$ additional lymphatic and/or non-lymphatic structures(s) relative to an RND (level VII, retropharyngeal lymph nodes, hypoglossal nerve) 
of increased chemoradiotherapy (CRT) use, with the need for less extensive surgery, there have been increased calls for further revisions due to some dissections not falling within distinct categories. ${ }^{18}$ There is conclusive evidence to suggest low long-term morbidity in selective compared to radical neck dissections (RND). ${ }^{19}$ Any surgical intervention must be validated by an accredited multidisciplinary team (MDT), comprising of members routinely involved in the delivery of care for head and neck cancer patients.

\section{Radiotherapy and Concurrent Chemotherapy}

Accredited departments are responsible for the delivery of megavoltage photons, with similar principles adopted as described for surgery. As the T stage increases, the likelihood of microscopic spread to other nodal groups also increases. As a result, a larger volume of tissue irradiation is required. ${ }^{2}$ Coventional radiotherapy (RT) consists of a once-daily treatment (fraction) over a 3-7 weeks period, with cumulative doses ranging from $50-70 \mathrm{~Gy} .{ }^{20}$ Whilst the morbidity associated with surgery is confined to the neck; RT has a multitude of side effects due to the field of irradiation, and often includes salivary glands and some viscera, resulting in debilitating mucositis and xerostomia. Conventional treatment techniques have now been replaced by intensity modulated radiotherapy (IMRT), and in particular for bilateral irradiation where the side effect profile is more significant. IMRT produces an even distribution of radiation dose within a target volume with irregular contours, subsequently sparing normal tissue in close proximity to a tumor. This provides scope for escalating radiation dose if required, ${ }^{20}$ as well as improving the $\mathrm{QOL}$, at least in the early stages of treatment. ${ }^{21}$

Following the publication of level 1 studies, there has been an upward trend in the use of primary and concomitant chemotherapy (CCT) ${ }^{22}$ Studies have demonstrated that both overall and progression-free survival in advanced head and neck cancers is improved, both in a primary and post-operative scenario, although morbidity is higher in patients who have previously undergone RND. ${ }^{2}$

\section{Management Strategies for Various Neck Nodal Stages}

Nodal treatment can either be elective, or therapeutic, in a clinically negative or positive neck respectively. The recent 8th edition of the AJCC has updated staging for non-p16+, non-EBER+, cancers of the head and neck nodal staging (Table 3) with the addition of extranodal extension (ENE) as a prognostic variable, in addition to number and size of metastatic lymph nodes. Determining unambiguous ENE will be challenging with current imaging modalities but should support clinical ENE defined by the invasion of skin, infiltration of musculature/dense tethering to the adjacent structure, dysfunction of cranial nerve, the brachial plexus, the sympathetic trunk or phrenic nerve. Pathological ENE has been defined as the extension of metastatic carcinoma from within the lymph node through the fibrous capsule and into surrounding connective tissue, regardless of the presence of stromal reaction. ${ }^{23}$

\section{Clinical Node Negative (N0) Neck (New Primary)}

The assessment and management of the N0 neck with regards to elective dissection versus watchful waiting continue to remain an area of debate. Detection rates of micrometastasis (metastasis $<2 \mathrm{~mm}$ in size) in lymph nodes through clinical and radiological correlation is poor. ${ }^{24}$ The rationale for elective treatment is due to the relatively high incidence of subclinical disease in elective RND specimens. Several retrospective series have demonstrated the presence of micrometastasis in up to $25 \%$ of patient ${ }^{25}$ based on histological evaluation of neck dissection specimens in patients deemed to be node negative (N0), guiding a risk-based approach in the adoption of prophylactic treatment of the neck (Flow chart 1).

During the 1990s, a period where RND was widely accepted as the only procedure for elective neck treatment, the risk-benefit analysis was undertaken through retrospective studies. These suggested prophylactic treatment of the neck in instances where risk of occult nodal metastasis

Table 3: $\mathrm{N}$ staging for cervical metastasis

\begin{tabular}{ll}
\hline$N$ category & Criteria \\
\hline Nx & Unable to assess regional nodes \\
N0 & No regional metastasis \\
N1 & Metastasis in a single ipsilateral node $\leq 3 \mathrm{~cm}$ in greatest dimension, ENE-negative \\
N2a & Metastasis in a single ipsilateral lymph $\geq 3$ and $\leq 6 \mathrm{~cm}$ in greatest dimension, ENE-negative \\
N2b & Metastasis in multiple ipsilateral lymph nodes, $\leq 6 \mathrm{~cm}$ in greatest dimension, ENE-negative \\
N2c & Metastasis in bilateral/contralateral lymph nodes, $\leq 6 \mathrm{~cm}$ in greatest dimension, ENE-negative \\
N3a & Metastasis in a lymph node $\geq 6 \mathrm{~cm}$ in greatest dimension, ENE-negative \\
\hline N3b & Metastasis in any node(s), ENE-positive \\
\hline ENE = extra-nodal extension
\end{tabular}


Flow chart 1: Algorithm for management of the NO neck (Paleri V, Urbano TG, Mehanna H, Repanos C, Lancaster J, Roques T, et al. Management of neck metastases in head and neck cancer: United Kingdom National Multidisciplinary Guidelines. J Laryngol Otol. 2016;130(S2):S161-S92)

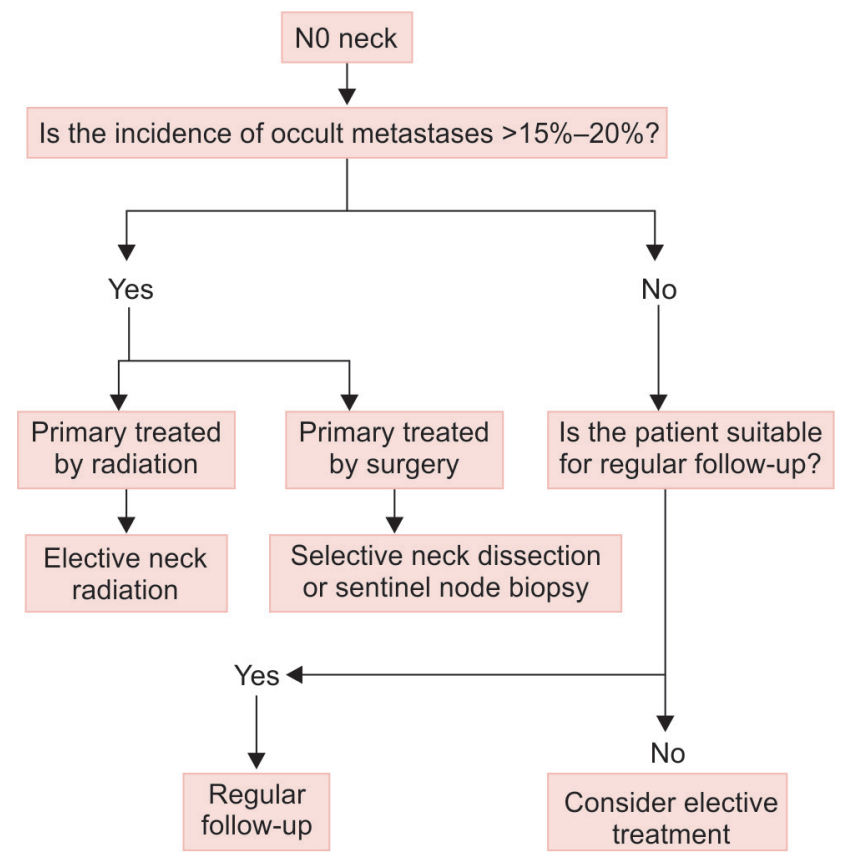

was $>20 \%$, although there has also been advocacy in those with a risk of $5-15 \%$ given the low morbidity associated with both treatment modalities. ${ }^{2}$ All SCC's of the upper aerodigestive tract, with the exception of T1-2 carcinomas of the glottis and select T1 cancers of the oropharynx would fall within the aforementioned category of an occult metastatic risk of $>15 \%$.

Protagonists of elective neck dissection cited lower relapse rates and better overall survival, ${ }^{26}$ whilst others considered the evidence to be non-definitive. ${ }^{27,28}$ Data from prospective trials further added to the conflicting evidence. ${ }^{29}$ An observational approach would have served to reduce an additional surgical procedure in $70 \%$ of patients who were deemed to be node-negative on histopathological evaluation, whilst providing simultaneous cost-benefit and reduced overall morbidity. ${ }^{30}$ Additionally, early nodal metastasis could be detected with the use of USS during the observational period, in the absence of compromised outcomes. ${ }^{29}$

Two recent major studies have attempted to address concerns in regards to the ideal management of the N0 neck in oral cavity SCC. D'Cruz et al., recently published the largest single-center RCT to date, comparing elective neck dissection (END) versus therapeutic neck dissection (TND) in 500 patients with T1/T2 N0 SCC of the oral cavity. At 3 years, END reported improved rate of overall survival compared to the TND group ( $80 \%$ vs. $67.5 \%$, $p=0.01)$ and disease-specific survival in the END than the TND group ( $45.9 \%$ vs $69.5 \%, p<0.001)$, with relapse within the TND presenting at a more advanced stage, with higher incidences of extracapsular spread. ${ }^{30}$ The transferability of the study findings have been questioned, as it only utilized ultrasound scan in the evaluation of the N0 neck where often now CT is preferred and the disproportionate use of adjuvant radiotherapy between the two groups (49\% END vs. 34\% TND) not included in the multivariate analysis.

Sentinel node biopsy (SNB) is not widely recognised as standard carein early oral and oropharyngeal cancer but does possess the ability to detect occult metastases in HNSCC, 31,32 through typical drainage patterns to echelon nodal basins depending upon the primary site. Particular promise has been seen in reducing unnecessary neck dissection (with consequent improved function, reduced morbidity ${ }^{33,34}$ and significantly reduced inpatient stay) $)^{35}$ allowing for comprehensive salvage surgery in a minimally disturbed surgical bed and identifying the need for unilateral versus bilateral neck dissection in midline tumours. ${ }^{3}$

The Sentinel European Node (SENT) trial, the largest observational prospective study to date incorporating fourteen European centres, recruited 415 patients with radiologically staged T1-T2 oral SCC. A sentinel node (SN) was detected in $99.5 \%$ and positive $\mathrm{SNs}$ were found in $23 \%$. False-negative result occurred in $14 \%$ ( $7 \%$ were amenable to a salvage procedure) with recurrence in 22 patients following a positive SNB and SND. Disease specific survival was $94 \%$ (at 3-years) with sensitivity of SNB of $86 \%$ and negative predictive value of $95 \%$. The low rate of regional recurrence within the study, counters the historical concerns of dissemination of tumour within the neck when biopsying suspected neck metastasis ${ }^{36,37}$ Of the 47 patients with midline tumors who would have otherwise undergone a bilateral neck dissection, 16 patients based on a positive $\mathrm{SN}$ went onto have a neck dissection of which only 8 required a bilateral neck dissection. The study suggests SNB to be both reliable and safe for staging the clinically N0 neck in T1/T2 oral cancers. However, as the study authors highlight, further evaluation through prospective RCTs is needed and refinement of the SNB technique will improve both ease and accuracy of sampling through future incorporation of intraoperative 3D navigation, ${ }^{38}$ new tracers ${ }^{39}$ and fluorescent markers. ${ }^{40}$

Currently, the choice of treatment at present for the N0 neck lies between modified radical neck dissection (MRND) and selective neck dissection (SND), with prospective studies demonstrating equal effectiveness of the SND compared to MRND, with reduced morbidity rates. ${ }^{2}$ There is no further role for the historically accepted RND in the treatment of the clinically N0 neck. ${ }^{41}$ SND of levels I-III should be undertaken in the case of oral tumors and levels II-IV in cases of oropharyngeal, laryngeal and hypopharyngeal SCCs. Level IIb dissection is seldom necessary for most 
patients with $\mathrm{N} 0$ disease, as metastasis to this subsite is $<2 \% .^{2}$ Clearance of this level during SND involves operating through a significantly narrow field, with marked retraction of the SCM. This will invariably result in traction injury of the accessory nerve, and a subsequent neuropraxia resulting in shoulder dysfunction for very little oncological benefit in laryngeal cancer (although slightly higher metastatic rates of 3.4\% are reported in the case of SCCs of the oral. ${ }^{42}$ There also remains a consensus in addressing level IV in oral SCCs due to the presence of skip lesions, in tongue base tumors, ${ }^{43}$ with reported rates of $2 \% .{ }^{44}$

Elective neck irradiation is as effective as elective neck dissection in the control of subclinical disease, with reported rates of $90 \% .{ }^{2}$ When irradiating the primary site, nodes deemed highest risk of harboring occult disease (the echelon lymph nodes), are also incorporated within the treatment field. Literature comparing elective ND and neck irradiation in tumors of the oral cavity, oropharynx, and larynx demonstrated no statistical difference in local control at 60 months. Local control was also statistically better in patients with hypopharyngeal cancer, treated with RT. ${ }^{2}$

In the case of a unilateral primary with ipsilateral nodal disease, historical data suggest a significantly high incidence of occult metastases within the contralateral neck, ranging between $30 \%$ and $70 \%$, especially in supraglottic, ${ }^{45,46}$ hypopharyngeal ${ }^{47,48}$ and oropharyngeal tumors. ${ }^{49}$ In advanced primaries, with a high risk of occult spread, contralateral neck treatment should be considered.

Large retrospective series data has also reported the risk of contralateral nodal involvement from a primary site, with contralateral neck treatment advocated in instances where the occult metastatic risk exceeds $20 \%$. This includes tumors encroaching or passing the midline. In instances where treatment of the bilateral neck is indicated, nodal irradiation may be preferred due to the reduced associated morbidity. ${ }^{2}$

The untreated N0 neck requires long-term surveillance, with early detection and treatment essential prior to disease becoming clinically detectable. Consideration should as such be directed to routine USS and FNAC. ${ }^{29}$ Despite routine surveillance, clinicians should be aware that patients within this cohort will present clinically with overt disease and require a therapeutic ND.

\section{Disease Recurrence at the Primary Site}

If the neck has been incorporated within the treatment field, occult metastatic rates remain as low as $5 \% .{ }^{2}$ Salvage ND is associated with greater rates of morbidity. As such, elective ND in the salvage setting may not be required if primary resection or reconstruction does not require access to major vessels.

\section{Node-positive Neck}

Active treatment is required in the instance of either clinical or radiologically positive nodal disease, with level 1 studies formulating algorithms to guide clinicians in the treatment of metastatic disease based on the treatment to the primary site, ${ }^{50}$ There is a high risk of occult metastasis to clinically uninvolved levels, and depending on the primary site, treatment to these regions may also be warranted. Level V is least likely to be involved, with studies showing positive nodes in up to $7 \%$ of patients undergoing a RND. ${ }^{2}$

\section{N1 Disease}

Data suggest that single modality therapy in ipsilateral solitary nodal disease (where nodes are $<3 \mathrm{~cm}$ in size) is adequate. In the instances where surgery is the modality of choice, SND may be adequate. ${ }^{2}$ Most patients will require postoperative RT given half of all N1 patients will have their nodal status upstaged following pathological evaluation. In the absence of bulky disease, SND with postoperative RT achieves local rates equivalent to comprehensive ND. ${ }^{41}$ An adequate response is demonstrated in patients with nodes $<3 \mathrm{~cm}$, with regional control rates following RT, improved in patients with nodes $<2 \mathrm{~cm}$. Concurrent CRT offers excellent control rates, ${ }^{51}$ with no further treatment warranted for complete responders, and an ND advocated in partial responders.

\section{N2a/b Disease}

A wide array of practice is associated with advanced nodal disease due to the lack of prospective RCTs. ${ }^{52}$ The treatment for this subset of patients is dependent on the management of the primary, and if deemed operable, a comprehensive neck dissection should be offered. If macroscopically clear of disease, an MRND with sparing of the accessory nerve achieves comparable control to an RND. ${ }^{53,54}$ Postoperative adjuvant treatment is recommended in larger nodes, given the risk of extranodal extension is higher.

With the increasing use of chemoradiotherapy in the primary, there was wide variation in practice in the management of advanced nodal disease (N2 or N3) as retrospective studies had shown persistent nodal disease in $40 \%$ of patients post chemoradiotherapy. ${ }^{55}$ As a result, the practice of planned neck dissection either before or after chemoradiation continued. ${ }^{52}$ The need for a neck dissection post chemoradiation has been addressed with the recent publication of the prospective PET-Neck RCT. 
The PET-neck phase III RCT ${ }^{22}$ compared PET-CT guided surveillance versus a planned ND for $\geq \mathrm{N} 2$ disease, where the primary treatment modality was CRT. This prospective trial, recruiting 282 patients to each treatment arm, demonstrated comparable survival outcomes in both p16 positive and negative patients, with a minimum followup period of 2 years. An active surveillance strategy with PET-CT led to fewer ND procedures in $80 \%$ of patients, and a subsequent reduction in morbidity, and a per patient cost saving of $£ 1415$. There were an additional 0.07 additional quality-adjusted life years (QALY) in contrast to elective ND. Hence, the current standard of care is a PET-CT scan 8 to 12 weeks following completion of CRT (due to false positive rates at an earlier stage), and elective neck dissection in those who demonstrate an incomplete response. Complete response on PET-CT requires no further treatment. ${ }^{13}$ The extent of salvage procedures is usually based on local guidance, although a trend is developing where limited clearance of involved levels or adjacent levels is undertaken. In instances of unresectable disease, RT or CRT is the sole modality available, and one must recognize the low likelihood of a curative outcome. ${ }^{2}$ In cases of small but inaccessible tumors, with advanced neck disease, resection of any nodal disease can be followed by RT +/- CT to a primary tumor and RT to the contralateral neck. There must be an appreciation that such a treatment strategy may result in a delay in treating the primary disease, and thus result in potential disease progression. . $^{213,56}$

\section{N3 Disease}

It is essential to accept that patients presenting with nodes $>6 \mathrm{~cm}$ will have some form of fixation to skin or underlying structures. Any decision to intervene surgically will be dependent on a multitude of factors including; the site of primary disease, presence/absence of fixation, structures involved in fixation, operator experience, and patient factors. Patients with N3 disease tend to present with distant metastatic disease, and PET-CT imaging is recommended to evaluate the extent of the disease fully.

Caution is advised in extrapolating the findings of PET-Neck trial to N3 (stage IVb), as this group formed a very small cohort $(n=17)$ within the study. Surgery in the N3 neck should always be in conjunction with CRT or RT alone, either pre- or postoperatively.

\section{Adjuvant Radiation for the Postoperative Neck}

Literature has quoted post-concurrent CRT complication rates in neck dissections to be as high as $28 \%{ }^{57}$ In the instance where the primary treatment modality is surgery (N2/3) neck disease, a RND is traditionally adopted. Disease control rates are comparable when RND or MRND is undertaken within a select group of patients. ${ }^{41}$
Controversies exist regarding carotid artery resection, particularly given extension into the adventitia occurs in $42 \%$ of patients, with luminal involvement rarely reported. ${ }^{58}$

Literature advocates the addition of RT postoperatively, to increase local control, and in particular, where adverse features are present (extracapsular spread, positive margins, $\geq \mathrm{T} 3$ disease, multiple nodes, level IV/V involvement, perineural invasion, vascular embolism). ${ }^{59}$ Randomized trials undertaken by the European Organisation for Treatment of Cancer (EORTC) and the Radiotherapy Oncology Group (RTOG), have demonstrated improved postoperative control with CCT in groups where margins are positive, or extracapsular spread has been detected.$^{60}$ In patients with $\geq 2$ lymph nodes as their only risk factor, in the absence of extra-capsular spread, did not benefit from the addition of chemotherapy. If the primary site is amenable to non-surgical intervention, the neck should be treated simultaneously. In the instances of $\geq \mathrm{N} 2$ disease, this will involve CRT.

\section{Human Papilloma Virus-associated Neck Disease}

A substantial amount of research has been undertaken in this distinct subset of head and neck cancer, which continues to increase in incidence within many parts of the world. ${ }^{61}$ The overwhelming majority of HPV associated cancer is observed within the oropharynx, and these patients tend to present with advanced disease, and extra-nodal extension. At present, no consensus exists as to whether HPV associated oropharyngeal squamous cell carcinoma (OPSCC) should be treated any differently to non-HPV HNSCC with nodal metastasis, despite better survival outcomes and results of de-escalation trials are eagerly awaited. The recent 8th edition of AJCC has now updated nodal staging in HPV associated OPSCC into both clinical and pathological stages (Table 4) reflecting that number rather than size and site of node impacted on survival.

Table 4: Clinical (c) and pathological ( $p$ ) nodal staging in HPV associated OPSCC

\begin{tabular}{ll}
\hline$N$ category $(c)$ & Criteria \\
\hline Nx & Unable to assess regional nodes \\
N0 & No regional \\
N1 & One or more ipsilateral nodes $\leq 6 \mathrm{~cm}$ in \\
& greatest dimension \\
N2 & Contralateralal or bilateral nodes $\leq 6 \mathrm{~cm}$ in \\
& greatest dimension \\
N3 & Lymph node $>6 \mathrm{~cm}$ \\
& \\
\hline$N$ category $(p)$ & Criteria \\
\hline Nx & Unable to assess regional nodes \\
N0 & No regional \\
N1 & Metastasis in $\leq 4$ lymph nodes \\
N2 & Metastasis in $>4$ lymph nodes \\
\hline
\end{tabular}




\section{Assessing Adequacy of Treatment}

Following RT, neck node size and fixity are good predictors of response rate and local control. In the case of N2 or N3 disease, there is a poor correlation between clinical and pathological response post-CRT. Owing to advances in cross-sectional imaging, recurrence rates as low as $10 \%$ have been found amongst the $30-45 \%$ of patients, who were deemed to have demonstrated a complete response following CRT. ${ }^{62,63}$ As a result, image-guided response-based approaches have gained prominence, without high-level evidence. Studies comparing CT with PET-CT demonstrated a higher efficacy of PET-CT in instances of advanced HNSCC and in particular those within a high-risk category with; a significant history of smoking, alcohol excess, or p16 -ve nonoropharyngeal malignancies. ${ }^{64}$ PET-CT has also been shown to be more costeffective $^{65}$ and accurate in identifying complete nodal response, particularly in p16 +ve patients $(93 \%$ vs. 50\% in standard CT). A planned PET-CT scan 10 to 12 weeks post CRT is now the accepted standard of care, with a negative scan indicative of an adequate response. High standardized uptake values (SUVs) are associated with residual disease and can be used as a decision-making tool for salvage surgery. ${ }^{13,14,56}$

\section{Management of Recurrent Disease of the Neck}

Any salvage surgery should be preceded by a thorough assessment to exclude distant metastasis. In the instances where recurrence has occurred following CRT and is deemed resectable, then surgical intervention through an oncologically adequate SND, with the understanding that associated morbidity is higher, is recommended. Where the disease is unresectable, re-irradiation may be considered, especially if the patient is $>2$ years after primary treatment. The introduction of IMRT and proton therapy results in significant sparing of critical neurological and vascular structures and means such treatment is more realistic when compared to previous regimes and modalities. Where recurrence follows primary surgical resection, re-resection combined with RT or primary RT +/- chemotherapy remain viable. ${ }^{2}$

\section{Palliative Care}

Patients with incurable nodal disease in the absence of distant metastasis present a significant clinical conundrum, given survival can extend for months and occasionally longer. Early involvement of the palliative care team plays a pivotal role in controlling the sequelae of advanced HNSCC, ${ }^{20}$ particularly before the development of associated complications. Fungating nodes can have a detrimental impact on psychosocial function, and implications on speech and swallowing require early speech and language therapy (SALT) and dietetic input to discuss the benefits and risks of overmedicalizing through enteral feeding. A role for palliative surgery, CCT (untreated HNSCC is usually chemosensitive, with response rates lower in recurrent disease), or RT, in improving patient quality of life exists in certain circumstances but should be used judiciously to avoid painful mucositis and subsequent distress with modest therapeutic gain. Overall prognosis, patient goals, morbidity, and likely benefits must be discussed so that expectations are realistic.

\section{REFERENCES}

1. Chaturvedi AK, Anderson WF, Lortet-Tieulent J, Curado MP, Ferlay J, Franceschi S, et al. Worldwide trends in incidence rates for oral cavity and oropharyngeal cancers. J Clin Oncol. 2013;31(36):4550-4559.

2. Paleri V, Urbano TG, Mehanna H, Repanos C, Lancaster J, Roques T, et al. Management of neck metastases in head and neck cancer: United Kingdom National Multidisciplinary Guidelines. J Laryngol Otol. 2016;130(S2):S161-S9.

3. Schilling C, Stoeckli SJ, Haerle SK, Broglie MA, Huber GF, Sorensen JA, et al. Sentinel European Node Trial (SENT): 3-year results of sentinel node biopsy in oral cancer. Eur J Cancer. 2015;51(18):2777-2784.

4. Liao LJ, Lo WC, Hsu WL, Wang CT, Lai MS. Detection of cervical lymph node metastasis in head and neck cancer patients with clinically N0 neck-a meta-analysis comparing different imaging modalities. BMC Cancer. 2012;12:236.

5. Van den Brekel MW, Stel HV, Castelijns JA, Nauta JJ, van der Waal I, Valk J, et al. Cervical lymph node metastasis: assessment of radiologic criteria. Radiology. 1990;177(2):379-384.

6. Shetty D, Jayade BV, Joshi SK, Gopalkrishnan K. Accuracy of palpation, ultrasonography, and computed tomography in the evaluation of metastatic cervical lymph nodes in head and neck cancer. Indian J Dent. 2015;6(3):121-124.

7. Merritt RM, Williams MF, James TH, Porubsky ES. Detection of cervical metastasis. A meta-analysis comparing computed tomography with physical examination. Arch Otolaryngol Head Neck Surg. 1997;123(2):149-152.

8. Blatt S, Ziebart T, Kruger M, Pabst AM. Diagnosing oral squamous cell carcinoma: How much imaging do we really need? A review of the current literature. J Craniomaxillofac Surg. 2016;44(5):538-549.

9. Richards PS, Peacock TE. The role of ultrasound in the detection of cervical lymph node metastases in clinically N0 squamous cell carcinoma of the head and neck. Cancer Imaging. 2007;7:167-178.

10. Tshering Vogel DW, Thoeny HC. Cross-sectional imaging in cancers of the head and neck: how we review and report. Cancer Imaging. 2016;16(1):20.

11. Amedee RG, Dhurandhar NR. Fine-needle aspiration biopsy. Laryngoscope. 2001;111(9):1551-1557.

12. Lyshchik A, Higashi T, Asato R, Tanaka S, Ito J, Hiraoka M, et al. Cervical lymph node metastases: diagnosis at sonoelastography--initial experience. Radiology. 2007;243(1):258-267.

13. Isles MG, McConkey C, Mehanna HM. A systematic review and meta-analysis of the role of positron emission tomography in the follow-up of head and neck squamous cell carcinoma following radiotherapy or chemoradiotherapy. Clin Otolaryngol. 2008;33(3):210-222. 
14. Gupta T, Master Z, Kannan S, Agarwal JP, Ghsoh-Laskar S, Rangarajan $V$, et al. Diagnostic performance of post-treatment FDG PET or FDG PET/CT imaging in head and neck cancer: a systematic review and meta-analysis. Eur J Nucl Med Mol Imaging. 2011;38(11):2083-2095.

15. Kyzas PA, Evangelou E, Denaxa-Kyza D, Ioannidis JP. 18F-fluorodeoxyglucose positron emission tomography to evaluate cervical node metastases in patients with head and neck squamous cell carcinoma: a meta-analysis. J Natl Cancer Inst. 2008;100(10):712-720.

16. Robson A. The management of the neck in squamous head and neck cancer. Clin Otolaryngol Allied Sci. 2001;26(2):157-161.

17. Robbins KT, Clayman G, Levine PA, Medina J, Sessions R, Shaha A, et al. Neck dissection classification update: revisions proposed by the American Head and Neck Society and the American Academy of Otolaryngology-Head and Neck Surgery. Arch Otolaryngol Head Neck Surg. 2002;128(7):751-758

18. Ferlito A, Robbins KT, Shah JP, Medina JE, Silver CE, Al-Tamimi S, et al. Proposal for a rational classification of neck dissections. Head Neck. 2011;33(3):445-450.

19. Harish K. Neck dissections: radical to conservative. World J Surg Oncol. 2005;3(1):21.

20. Sanderson RJ, Ironside JA. Squamous cell carcinomas of the head and neck. BMJ. 2002;325(7368):822-827.

21. Pow EH, Kwong DL, McMillan AS, Wong MC, Sham JS, Leung LH, et al. Xerostomia and quality of life after intensitymodulated radiotherapy vs. conventional radiotherapy for early-stage nasopharyngeal carcinoma: initial report on a randomized controlled clinical trial. Int J Radiat Oncol Biol Phys. 2006;66(4):981-991.

22. Mehanna H, Wong WL, McConkey CC, Rahman JK, Robinson M, Hartley AG, et al. PET-CT Surveillance versus Neck Dissection in Advanced Head and Neck Cancer. N Engl J Med. 2016;374(15):1444-1454.

23. Lydiatt WM, Patel SG, O'Sullivan B, Brandwein MS, Ridge JA, Migliacci JC et al. Head and Neck cancers-major changes in the American Joint Committee on cancer eighth edition cancer staging manual. CA Cancer J Clin. 2017 Mar;67(2):122-137.

24. Ambrosch P, Brinck U. Detection of nodal micrometastases in head and neck cancer by serial sectioning and immunostaining. Oncology (Williston Park). 1996;10(8):1221-1226; discussion 6,9 .

25. van den Brekel MW, van der Waal I, Meijer CJ, Freeman JL, Castelijns JA, Snow GB. The incidence of micrometastases in neck dissection specimens obtained from elective neck dissections. Laryngoscope. 1996;106(8):987-991.

26. Huang SF, Chang JT, Liao CT, Kang CJ, Lin CY, Fan KH, et al. The role of elective neck dissection in early stage buccal cancer. Laryngoscope. 2015;125(1):128-133.

27. Kramer D, Durham JS, Jackson S, Brookes J. Management of the neck in N0 squamous cell carcinoma of the oral cavity. J Otolaryngol. 2001;30(5):283-288.

28. D'Cruz AK, Siddachari RC, Walvekar RR, Pantvaidya GH, Chaukar DA, Deshpande MS, et al. Elective neck dissection for the management of the N0 neck in early cancer of the oral tongue: need for a randomized controlled trial. Head Neck. 2009;31(5):618-624.

29. Yuen AP, Ho CM, Chow TL, Tang LC, Cheung WY, Ng RW, et al. Prospective randomized study of selective neck dissection versus observation for N0 neck of early tongue carcinoma.
Head Neck. 2009;31(6):765-772.

30. D'CruzAK, Vaish R, Kapre N, Dandekar M, Gupta S, Hawaldar $R$, et al. Elective versus Therapeutic Neck Dissection in NodeNegative Oral Cancer. N Engl J Med. 2015;373(6):521-529.

31. Thompson CF, St John MA, Lawson G, Grogan T, Elashoff D, Mendelsohn AH. Diagnostic value of sentinel lymph node biopsy in head and neck cancer: a meta-analysis. Eur Arch Otorhinolaryngol. 2013;270(7):2115-2122.

32. Civantos FJ, Zitsch RP, Schuller DE, Agrawal A, Smith RB, Nason R, et al. Sentinel lymph node biopsy accurately stages the regional lymph nodes for T1-T2 oral squamous cell carcinomas: results of a prospective multi-institutional trial. J Clin Oncol. 2010;28(8):1395-400.

33. Schiefke F, Akdemir M, Weber A, Akdemir D, Singer S, Frerich B. Function, postoperative morbidity, and quality of life after cervical sentinel node biopsy and after selective neck dissection. Head Neck. 2009;31(4):503-512.

34. Murer K, Huber GF, Haile SR, Stoeckli SJ. Comparison of morbidity between sentinel node biopsy and elective neck dissection for treatment of the n0 neck in patients with oral squamous cell carcinoma. Head Neck. 2011;33(9):1260-1264.

35. O'Connor R, Pezier T, Schilling C, McGurk M. The relative cost of sentinel lymph node biopsy in early oral cancer. J Craniomaxillofac Surg. 2013;41(8):721-727.

36. Brown JS, Shaw RJ, Bekiroglu F, Rogers SN. Systematic review of the current evidence in the use of postoperative radiotherapy for oral squamous cell carcinoma. Br J Oral Maxillofac Surg. 2012;50(6):481-489.

37. Ganly I, Goldstein D, Carlson DL, Patel SG, O'Sullivan B, Lee $\mathrm{N}$, et al. Long-term regional control and survival in patients with "low-risk," early stage oral tongue cancer managed by partial glossectomy and neck dissection without postoperative radiation: the importance of tumor thickness. Cancer. 2013;119(6):1168-1176.

38. Heuveling DA, van Weert S, Karagozoglu KH, de Bree R. Evaluation of the use of freehand SPECT for sentinel node biopsy in early stage oral carcinoma. Oral Oncol. 2015;51(3):287-290.

39. Agrawal A, Civantos FJ, Brumund KT, Chepeha DB, Hall NC, Carroll WR, et al. [(99m)Tc]Tilmanocept Accurately Detects Sentinel Lymph Nodes and Predicts Node Pathology Status in Patients with Oral Squamous Cell Carcinoma of the Head and Neck: Results of a Phase III Multi-institutional Trial. Ann Surg Oncol. 2015;22(11):3708-3715.

40. Brouwer OR, Klop WM, Buckle T, Vermeeren L, van den Brekel MW, Balm AJ, et al. Feasibility of sentinel node biopsy in head and neck melanoma using a hybrid radioactive and fluorescent tracer. Ann Surg Oncol. 2012;19(6):1988-1994.

41. O'Hara J, Simo R, McQueen A, Andi K, Lester S, Giddings C, et al. Management of metastatic neck disease--summary of the 11th Evidence Based Management Day. Clin Otolaryngol. 2014;39(1):3-5.

42. de Vicente JC, Rodriguez-Santamarta T, Pena I, Villalain L, Fernandez-Valle A, Gonzalez-Garcia M. Relevance of level IIb neck dissection in oral squamous cell carcinoma. Med Oral Patol Oral Cir Bucal. 2015;20(5):e547-553.

43. Dass A, Singhal SK, Punia R, Gupta N, Verma H, Budhiraja S, et al. Role of Neck Dissection in Clinical T3NOM0 Lesion of Oral Cavity: Changing Trend. J Clin Diagn Res. 2017;11(8):XC12-XC4.

44. Dias FL, Lima RA, Kligerman J, Farias TP, Soares JR, Manfro $\mathrm{G}$, et al. Relevance of skip metastases for squamous cell carcinoma of the oral tongue and the floor of the mouth. Otolaryngol Head Neck Surg. 2006;134(3):460-465. 
45. Redaelli de Zinis LO, Nicolai P, Tomenzoli D, Ghizzardi D, Trimarchi M, Cappiello J, et al. The distribution of lymph node metastases in supraglottic squamous cell carcinoma: therapeutic implications. Head Neck. 2002;24(10):913-920.

46. Hicks WL, Jr., Kollmorgen DR, Kuriakose MA, Orner J, Bakamjian VY, Winston J, et al. Patterns of nodal metastasis and surgical management of the neck in supraglottic laryngeal carcinoma. Otolaryngol Head Neck Surg. 1999;121(1):57-61.

47. Aluffi P, Pisani P, Policarpo M, Pia F. Contralateral cervical lymph node metastases in pyriform sinus carcinoma. Otolaryngol Head Neck Surg. 2006;134(4):650-653.

48. Koo BS, Lim YC, Lee JS, Kim YH, Kim SH, Choi EC. Management of contralateral N0 neck in pyriform sinus carcinoma. Laryngoscope. 2006;116(7):1268-1272.

49. Lim YC, Koo BS, Lee JS, Lim JY, Choi EC. Distributions of cervical lymph node metastases in oropharyngeal carcinoma: therapeutic implications for the N0 neck. Laryngoscope. 2006;116(7):1148-1152.

50. Iyer NG, Tan DS, Tan VK, Wang W, Hwang J, Tan NC, et al. Randomized trial comparing surgery and adjuvant radiotherapy versus concurrent chemoradiotherapy in patients with advanced, nonmetastatic squamous cell carcinoma of the head and neck: 10-year update and subset analysis. Cancer. 2015;121(10):1599-1607.

51. Moore MG, Bhattacharyya N. Effectiveness of chemotherapy and radiotherapy in sterilizing cervical nodal disease in squamous cell carcinoma of the head and neck. Laryngoscope. 2005;115(4):570-573.

52. Thariat J, Ang KK, Allen PK, Ahamad A, Williams MD, Myers JN, et al. Prediction of neck dissection requirement after definitive radiotherapy for head-and-neck squamous cell carcinoma. Int J Radiat Oncol Biol Phys. 2012;82(3):e367-374.

53. Richards BL, Spiro JD. Controlling advanced neck disease: efficacy of neck dissection and radiotherapy. Laryngoscope. 2000;110(7):1124-1127.

54. Andersen PE, Shah JP, Cambronero E, Spiro RH. The role of comprehensive neck dissection with preservation of the spinal accessory nerve in the clinically positive neck. Am J Surg. 1994;168(5):499-502.

55. Stenson KM, Haraf DJ, Pelzer H, Recant W, Kies MS, Weichselbaum RR, et al. The role of cervical lymphadenectomy after aggressive concomitant chemoradiotherapy: the feasibility of selective neck dissection. Arch Otolaryngol Head Neck Surg. 2000;126(8):950-956.

56. Wee JT, Anderson BO, Corry J, D'Cruz A, Soo KC, Qian CN, et al. Management of the neck after chemoradiotherapy for head and neck cancers in Asia: consensus statement from the Asian Oncology Summit 2009. Lancet Oncol. 2009;10(11):1086-1092.

57. van den Bovenkamp K, Noordhuis MG, Oosting SF, van der Laan B, Roodenburg JL, Bijl HP, et al. Clinical outcome of salvage neck dissections in head and neck cancer in relation to initial treatment, extent of surgery and patient factors. Clin Otolaryngol. 2017;42(3):693-700.

58. Huvos AG, Leaming RH, Moore OS. Clinicopathologic study of the resected carotid artery. Analysis of sixty-four cases. Am J Surg. 1973;126(4):570-574.

59. Pignon JP, Bourhis J, Domenge C, Designe L. Chemotherapy added to locoregional treatment for head and neck squamous-cell carcinoma: three meta-analyses of updated individual data. MACH-NC Collaborative Group. MetaAnalysis of Chemotherapy on Head and Neck Cancer. Lancet. 2000;355(9208):949-955.

60. Cooper JS, Zhang Q, Pajak TF, Forastiere AA, Jacobs J, Saxman SB, et al. Long-term follow-up of the RTOG 9501/ intergroup phase III trial: postoperative concurrent radiation therapy and chemotherapy in high-risk squamous cell carcinoma of the head and neck. Int J Radiat Oncol Biol Phys. 2012;84(5):1198-1205.

61. Ramqvist T, Dalianis T. Oropharyngeal cancer epidemic and human papillomavirus. Emerg Infect Dis. 2010;16(11):1671-1677.

62. Hanna E, Alexiou M, Morgan J, Badley J, Maddox AM, Penagaricano J, et al. Intensive chemoradiotherapy as a primary treatment for organ preservation in patients with advanced cancer of the head and neck: efficacy, toxic effects, and limitations. Arch Otolaryngol Head Neck Surg. 2004;130(7):861-867.

63. Liauw SL, Mancuso AA, Amdur RJ, Morris CG, Villaret DB, Werning JW, et al. Postradiotherapy neck dissection for lymph node-positive head and neck cancer: the use of computed tomography to manage the neck. J Clin Oncol. 2006;24(9):1421-1427.

64. Moeller BJ, Rana V, Cannon BA, Williams MD, Sturgis EM, Ginsberg LE, et al. Prospective risk-adjusted [18F] Fluorodeoxyglucose positron emission tomography and computed tomography assessment of radiation response in head and neck cancer. J Clin Oncol. 2009;27(15):2509-2515.

65. Pryor DI, Porceddu SV, Scuffham PA, Whitty JA, Thomas PA, Burmeister BH. Economic analysis of FDG-PET-guided management of the neck after primary chemoradiotherapy for node-positive head and neck squamous cell carcinoma. Head Neck. 2013;35(9):1287-1294. 